\title{
The magnetic fields of the quiet solar photosphere
}

\author{
Nataliya G. Shchukina ${ }^{1}$ and Javier Trujillo Bueno ${ }^{2}$ \\ ${ }^{1}$ Main Astronomical Observatory, National Academy of Sciences, 03680, Kyiv, Ukraine email: \\ shchukin@mao.kiev.ua \\ ${ }^{2}$ Instituto de Astrofísica de Canarias, 38205 La Laguna, Tenerife, Spain email: jtb@iac.es
}

\begin{abstract}
We report on a novel investigation of the complex magnetism of the quiet Sun which is based on a realistic three-dimensional modeling of the Hanle effect.
\end{abstract}

\section{Introduction}

Investigations on the magnetism of the quiet Sun require the application of polarized radiation diagnostics techniques that take proper account of the possibility of magnetic structuring well beyond the spatial resolution limit. Diagnostic methods based on the Zeeman effect have given us a wealth of information on the complex magnetism of the quiet Sun, but concerning only $\sim 1 \%$ of the photosphere volume. In our opinion, the challenge continues being the following: how to investigate the magnetism of the remaining $\sim 99 \%$ of the quiet solar photosphere ? The Hanle effect (Hanle 1924) has the required potential (Stenflo 1982). Here we present results of a new approach to the Hanle effect based on realistic multi-level and three-dimensional radiative transfer modeling of the observed linear scattering polarization in the Sr I $\lambda 4607$ line.

\section{The $1 \mathrm{D}$ versus the $3 \mathrm{D}$ approach}

The first calculations of the scattering polarization in the Sr I $\lambda 4607$ line were carried out by Faurobert-Scholl and collaborators $(1993,1995,2001)$ assuming a two-level atomic model and that the dynamic and highly inhomogeneous solar photosphere can be well represented by a one-dimensional (1D) semi-empirical model. Such an approach uses ad-hoc fitting parameters (microturbulence \& macroturbulence) to model the emergent spectral line radiation. Recently we have shown (Shchukina \& Trujillo Bueno 2003) that the $1 \mathrm{D}$ strategy is unreliable.

We contrast here the 1D modelling with a new approach based on three-dimensional (3D) scattering polarization calculations in snapshot taken from realistic radiation hydrodynamical simulations of solar surface convection (Asplund et al. 2000). Our 1D modeling of the observed scattering polarization in the strontium line have been carried out using the FAL-C atmospheric model of Fontenla et al. (1993), taking into account the model's microturbulent velocity, but using the macroturbulent velocity as a free parameter.

In our calculations we have considered multilevel atomic model. It has 15 levels for Sr I plus 1 level for Sr II. 


\section{Results and conclusions}

The emergent Stokes profiles calculated both in the 1D and 3D models of the solar photosphere give a spatially-averaged fractional linear polarization $Q / I$ that is larger than the observed $Q / I$, thus indicating the need for invoking magnetic depolarization.

The macroturbulent velocity is a free parameter that has a serious impact on the calculated polarization amplitudes and hence on the value of the inferred magnetic field strength $B$. 1D approach yields artificially low values of $B$ : between $\sim 10$ and $\sim 30 \mathrm{G}$.

Our 3D modelling approach takes fully into account the effect of the hydrodynamical flow velocities. As a result the synthetic intensity Stokes- $I$ profiles are automatically in excellent agreement with the observations - that is without having to use any adhoc fitting parameters like microturbulence or macroturbulence (for more details see Shchukina \& Trujillo Bueno 2003).

For the simplest model of a volume-filling single-valued microturbulent magnetic field, our 3D simulations of the Hanle effect for $\langle B\rangle \sim 60 \mathrm{G}$ produces a notable agreement with the observed spatially and temporally $Q / I$. We point out that the strontium line is sensitive to the Hanle effect between 1 and $300 \mathrm{G}$, approximately.

Recent MHD simulations (Cattaneo 1999, Stein \& Nordlund 2003) and observations of the Zeeman effect in the inrared $1.56 \mu \mathrm{m}$ Fe I lines (Khomenko et al. 2003) suggest that the photospheric plasma of the quiet Sun must have a continuous distribution of field strengths with a Probability Distribution Function (PDF) whose shape is close to exponential. If we assume that $\mathrm{PDF}=\left(1 / B_{0}\right) \exp \left(-B / B_{0}\right)$ for both the granular and intergranular regions, we then find that $B_{0}=130 \mathrm{G}$ yields a fairy good fit to the observed $Q / I$. This implies that in the quiet Sun $\langle B\rangle=130 \mathrm{G}$.

For a more detailed presentation including also results of an investigation of the Hanle effect in molecular lines and with a detailed discussion of the implications of our results see Trujillo Bueno, Shchukina, \& Asensio Ramos (2004).

\section{Acknowledgements}

This research has been supported by the INTAS project 00-00084 and by the Spanish Plan Nacional de Astronomía y Astrofísica through project AYA2001-1649.

\section{References}

Asplund, M., Nordlund, Å., Trampedach, R., Allende Prieto, C. \& Stein, R.F. 2000 Astron. Astrophys. 359, 729-742.

Cattaneo, F. 1999 Astrophys. J. 515, L39-L42.

Faurobert-Scholl, M., Feautrier, N., Machefert, F., Petrovay, K. \& Spielfiedel, A. 2001 Astron. Astrophys. 378, 627-634.

Fontenla, J. M., Avrett, E. \& Loeser, R. 1993 Astrophys. J. 406, 319-345.

Hanle, W. 1924 Z. Phys. 30, 93-105.

Khomenko, E., Collados, M., Solanki, S.K., Lagg, A. \& Trujillo Bueno, J. 2003 Astron. Astrophys. 408, 1115-1135.

Shchukina, N., \& Trujillo Bueno, J. 2003 in Solar Polarization 3, (eds J. Trujillo Bueno \& J. Sanchez Almeida). ASP Conf. Ser. Vol. 307, pp. 336-343, Astronomical Society of Pacific, San Francisco.

Stein, R.F. \& Nordlund, A. 2003 in Modelling of Stellar Atmospheres, (eds N.E. Piskunov, W.W. Weiss \& D.F. Gray). ASP Conf. Ser. Vol. IAU 210, pp. 169-180, Astronomical Society of Pacific, San Francisco.

Stenflo, J. 1982 Solar Phys. 80, 209-226.

Trujillo Bueno, J., Shchukina, N. \& Asensio Ramos, A. 2004 Nature 430, 326-329. 